Article

\title{
Theoretical and Experimental Electrostatic Potential around the m-Nitrophenol Molecule
}

\author{
Mokhtaria Drissi, Nadia Benhalima, Youcef Megrouss, Rahmani Rachida, Abdelkader Chouaih \\ and Fodil Hamzaoui *
}

Laboratoire LTPS, Faculté des Sciences et de la Technologie, Université de Mostaganem, 27000-Mostaganem, Algeria; E-Mails: mokadrissi@gmail.com (M.D.); nadiallotus@yahoo.fr (N.B.); youmeg@hotmail.fr (Y.M.); rahmani_ra63@yahoo.fr (R.R.); aek_chouaih@yahoo.fr (A.C.)

* Author to whom correspondence should be addressed; E-Mail: Fodil_hamzaoui@yahoo.fr; Tel.: +213-45-33-34-88; Fax: +213-45-33-13-69.

Academic Editor: Derek J. McPhee

Received: 22 December 2014 / Accepted: 25 February 2015 / Published: 3 March 2015

\begin{abstract}
This work concerns a comparison of experimental and theoretical results of the electron charge density distribution and the electrostatic potential around the $m$-nitrophenol molecule (m-NPH) known for its interesting physical characteristics. The molecular experimental results have been obtained from a high-resolution X-ray diffraction study. Theoretical investigations were performed using the Density Functional Theory at B3LYP level of theory at $6-31 \mathrm{G}^{*}$ in the Gaussian program. The multipolar model of Hansen and Coppens was used for the experimental electron charge density distribution around the molecule, while we used the DFT methods for the theoretical calculations. The electron charge density obtained in both methods allowed us to find out different molecular properties such us the electrostatic potential and the dipole moment, which were finally subject to a comparison leading to a good match obtained between both methods. The intramolecular charge transfer has also been confirmed by an HOMO-LUMO analysis.
\end{abstract}

Keywords: electron charge density; $m$-nitrophenol; nonlinear optical compound (NLO); electrostatic potential; optimized geometry; HOMO-LUMO 


\section{Introduction}

$m$-Nitrophenol (m-NPH) occurs in two polymorphic forms: orthorhombic (P2 $\left.122_{1} 2_{1}\right)$ and monoclinic (P2 $1 / n$ ) (see Figure 1). We are going to concentrate on the monoclinic form as the first form has already been the subject of a preceding report [1]. The main purpose of our work is to establish the electrostatic potential around the molecule through the determination of the electron charge density. This electrostatic potential will help us to describe and understand the inter- and intramolecular interactions (charge transfer) in the crystal. The presented electrostatic potential can be a starting point for the estimation of crystal energy cohesion in order to get more information about the existence of the polymorphism in the compound $m$-nitrophenol [2-4].

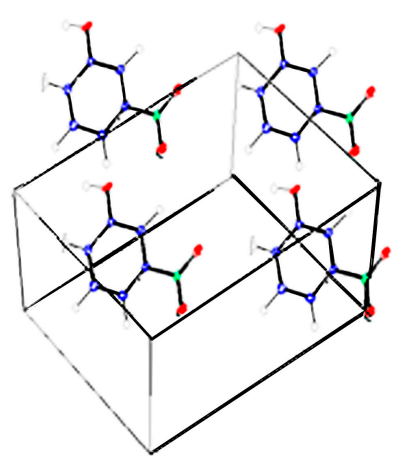

(a)

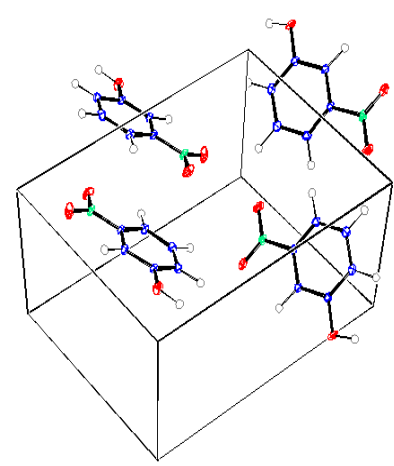

(b)

Figure 1. Polymorphic forms of $m$-nitrophenol, (a) orthorhombic, (b) monoclinic.

We have previously published an article about the high resolution X-ray diffraction and crystallographic study with a thermal motion analysis of the compound m-NPH [5]. The lasting metastability of the monoclinic form of $m$-nitrophenol originates probably from interactions within the centrosymmetric dimers of overlapping molecules. The attractive interactions within centrosymmetric dimers of overlapping molecules are as strong as interactions between hydrogen-bonded molecules [6,7]. We also presented the molecular dipole moment based on spherical model refinement [8]. In the present work, we based our study on the multipolar model of Coppens-Hansen where the non-spherical aspect of the atoms was taken into account. The multipole model represents an extrapolation to infinite resolution from a finite set of experimental data [9]. This last refinement gave us an accurate picture of the electronic charge density distribution in the compound m-NPH.

The theoretical part adds to our experimental work by using ab-initio calculations through providing a comparison of the molecular electrostatic properties such as the dipole moment, the electron density maps and the electrostatic potential with the experimental data.

\section{Experimental Details}

The accurate electron density distribution and the electrostatic potential around the molecule (m-NPH) have been calculated from a high-resolution X-ray diffraction study [5]. A summarized table of the X-ray experiment details of the crystallographic data is given in Table 1. 
Table 1. Experimental details.

\begin{tabular}{cc}
\hline Crystal Data & \\
\hline Chemical formula & $\mathrm{C}_{6} \mathrm{H}_{5} \mathrm{NO}_{3}$ \\
Chemical formula weight & 139.11 \\
Cell setting & Monoclinic \\
Space group & $\mathrm{P} 2_{1} / n$ \\
$a(\AA)$ & $11.026(4)$ \\
$b(\AA)$ & $6.736(1)$ \\
$c(\AA)$ & $8.119(21)$ \\
$\beta\left({ }^{\circ}\right)$ & $97.73(2)$ \\
$V\left(\AA^{3}\right)$ & 597.50 \\
$Z$ & 4 \\
Radiation type & Mo $K \alpha$ \\
Temperature $(\mathrm{K})$ & $122(1)$ \\
No. of measured reflections & 3148 \\
\hline
\end{tabular}

The Hansen-Coppens multipole formalism [10], as implemented in the MOPRO least squares program [11] for multipole refinement, was used for both observed and theoretical structure factor fitting. This package is based on program of least square method using non spherical electron distribution around the atoms $[12,13]$.

The rigid pseudo-atom model Hansen-Coppens is commonly used in analysis of the charge density distribution. The electron density $\rho(\vec{r})$ in the crystal is described by a sum of so-called aspherical pseudo-atoms with nuclear positions $\overrightarrow{r_{k}}$ :

$$
\rho(\vec{r})=\sum_{k} \rho_{k}\left(\vec{r}-\overrightarrow{r_{k}}-u\right) * t_{k}(u)
$$

where $t_{k}(u)$ is a Gaussian thermal-displacement distribution and * indicates a convolution product. The different atomic densities are described as a series expansion in real spherical harmonic functions $Y_{l m}$ up to order four:

$$
\rho_{\text {atom }}(\vec{r})=\rho_{c}(\vec{r})+P_{v} K^{\prime 3} \rho_{v}\left(K^{\prime} \vec{r}\right)+\sum_{l=0}^{4} \sum_{m=-l}^{+l} K^{\prime \prime} R_{l}\left(K^{\prime \prime} \vec{r}\right) P_{l m} Y_{l m}\left(\frac{\vec{r}}{r}\right)
$$

In Equation (2), $\rho_{c}$ and $\rho_{v}$ are spherically averaged Hartree-Fock core and valence densities, with $\rho_{v}$ normalized to one electron, $Y_{l m}$ are multipolar spherical harmonic angular functions in real form and $R_{l}$ are Slater-type radial functions.

Two charge-density variables, $P_{v}$ and $P_{l m}$, the population parameters, and $K^{\prime}$ and $K^{\prime \prime}$ parameters which allows expansion and contraction of the valence shell, are added to the conventional structural analysis parameters [14]. The population parameters $P_{v}$ and $P_{l m}$ are floated along with $K^{\prime}$ and $K^{\prime \prime}$ during during the refinement. To reduce the number of variables, atoms having the same environment were assumed to have the same electron charge deformation: All hydrogen atoms were assumed to be equivalent and are described by the same Slater radial function. Also, the two oxygen atoms are assumed to have the same local symmetry. 
The least-square refinements method allowed us to the accurately get the net atomic charge, the molecular dipole moment and the electrostatic potential around the molecule. We have also described the electron density distribution in the crystal form.

\section{Computational Details}

The theoretical calculations were performedusingthe Density Functional Theory at B3LYP (Becke's three parameter hybrid functional using the correlation functional of Lee, Yang, and Parr, which includes both local and non-local terms correlation functional) methods at 6-31G* level [15]. To perform this computational work, we used the Gaussian 09 program package [16]. The Highest Occupied Molecular Orbital (HOMO)-Lowest Unoccupied Molecular Orbital (LUMO) analysis has been carried out to explain the charge transfer place within the molecule. The chemical hardness and chemical potential are also calculated using the HOMO and LUMO. The visualization of the electron charge and the electrostatic potential of the molecule were obtained using the Molden program [17].

\section{Results and Discussion}

\subsection{Optimization of Geometrical Parameters}

Geometry optimization is a name for the procedure that attempts to find the configuration of minimum energy of the molecule. The procedure calculates the wave function and the energy at a starting geometry and then proceeds to search a new geometry of a lower energy.

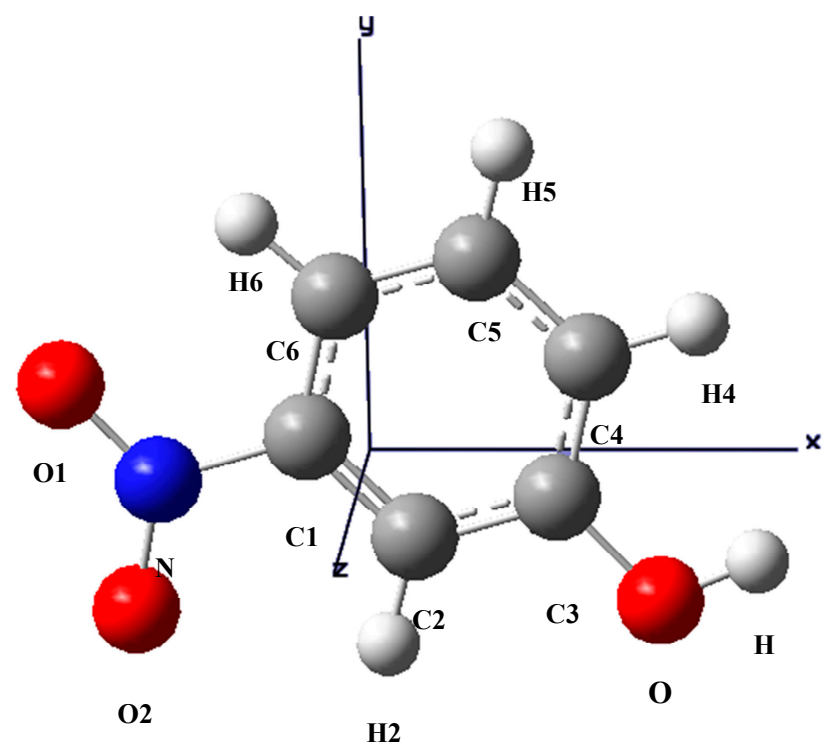

Figure 2. The optimized structure of m-NPH based on DFT B3LYP/6-1G* basis set.

The optimized structure of the title compound is shown in Figure 2. The calculated structure parameters (bond lengths, bond angles and torsion angles) were listed in Tables 2-4 where it can be seen that all the calculated parameters are in line with the X-ray results. In summary, the optimized bond lengths and bond angles obtained using the DFT method are in good agreement with the corresponding 
X-ray structural parameters. The calculated geometric parameters represent a good approximation and can provide a starting point to calculate other parameters, such as vibrational wavenumbers.

Table 2. Selected bond distances $(\AA)$ by X-ray and theoretical calculations (B3LYP/6-31G*).

\begin{tabular}{|c|c|c|c|}
\hline \multirow{2}{*}{ Atom 1} & \multirow{2}{*}{ Atom 2} & \multicolumn{2}{|c|}{ Distance $(\AA)$} \\
\hline & & X-ray & B3LYP/6-31G* \\
\hline $\mathrm{C} 1$ & C6 & 1.410 & 1.433 \\
\hline $\mathrm{C} 1$ & $\mathrm{C} 2$ & 1.396 & 1.384 \\
\hline $\mathrm{C} 2$ & $\mathrm{C} 3$ & 1.402 & 1.396 \\
\hline $\mathrm{C} 3$ & $\mathrm{C} 4$ & 1.411 & 1.392 \\
\hline $\mathrm{C} 4$ & $\mathrm{C} 5$ & 1.396 & 1.391 \\
\hline C6 & $\mathrm{C} 5$ & 1.400 & 1.412 \\
\hline $\mathrm{O}$ & $\mathrm{C} 3$ & 1.365 & 1.380 \\
\hline $\mathrm{C} 1$ & $\mathrm{~N}$ & 1.474 & 1.468 \\
\hline $\mathrm{O} 1$ & $\mathrm{~N}$ & 1.244 & 1.281 \\
\hline $\mathrm{O} 2$ & $\mathrm{~N}$ & 1.243 & 1.283 \\
\hline $\mathrm{O}$ & $\mathrm{H}$ & 1.030 & 0.992 \\
\hline H6 & C6 & 1.089 & 1.084 \\
\hline $\mathrm{H} 2$ & $\mathrm{C} 2$ & 1.085 & 1.078 \\
\hline H4 & $\mathrm{C} 4$ & 1.078 & 1.069 \\
\hline H5 & $\mathrm{C} 5$ & 1.085 & 1.082 \\
\hline
\end{tabular}

Table 3. Selected bond angles $\left(^{\circ}\right)$ by X-ray and theoretical calculations (B3LYP/6-31G*).

\begin{tabular}{ccccc}
\hline \multirow{2}{*}{ Atom 1 } & \multirow{2}{*}{ Atom 2 } & \multirow{2}{*}{ Atom 3 } & \multicolumn{2}{c}{ Angle $\left(^{(}\right)$} \\
\cline { 4 - 5 } & & & X-ray & B3LYP/6-31G* \\
\hline C6 & $\mathrm{C} 1$ & $\mathrm{~N}$ & 118.90 & 118.46 \\
$\mathrm{C} 6$ & $\mathrm{C} 1$ & $\mathrm{C} 2$ & 124.11 & 122.55 \\
$\mathrm{~N}$ & $\mathrm{C} 1$ & $\mathrm{C} 2$ & 116.99 & 117.37 \\
$\mathrm{H} 2$ & $\mathrm{C} 2$ & $\mathrm{C} 3$ & 122.50 & 119.45 \\
$\mathrm{H} 2$ & $\mathrm{C} 2$ & $\mathrm{C} 1$ & 120.51 & 120.82 \\
$\mathrm{C} 3$ & $\mathrm{C} 2$ & $\mathrm{C} 1$ & 118.99 & 119.10 \\
$\mathrm{C} 4$ & $\mathrm{C} 3$ & $\mathrm{O}$ & 123.31 & 122.72 \\
$\mathrm{H} 4$ & $\mathrm{C} 4$ & $\mathrm{C} 3$ & 120.31 & 119.88 \\
$\mathrm{H} 4$ & $\mathrm{C} 4$ & $\mathrm{C} 5$ & 118.98 & 118.94 \\
$\mathrm{C} 3$ & $\mathrm{C} 4$ & $\mathrm{C} 5$ & 120.71 & 118.06 \\
$\mathrm{H} 5$ & $\mathrm{C} 5$ & $\mathrm{C} 6$ & 119.20 & 119.87 \\
$\mathrm{H} 5$ & $\mathrm{C} 5$ & $\mathrm{C} 4$ & 120.66 & 119.74 \\
$\mathrm{C} 6$ & $\mathrm{C} 5$ & $\mathrm{C} 4$ & 120.14 & 119.28 \\
$\mathrm{H} 6$ & $\mathrm{C} 6$ & $\mathrm{C} 5$ & 119.39 & 120.13 \\
$\mathrm{H} 6$ & $\mathrm{C} 6$ & $\mathrm{C} 1$ & 123.00 & 120.91 \\
$\mathrm{C} 5$ & $\mathrm{C} 6$ & $\mathrm{C} 1$ & 117.60 & 119.28 \\
$\mathrm{H}$ & $\mathrm{O}$ & $\mathrm{C} 3$ & 109.00 & 110.55 \\
O2 & $\mathrm{N}$ & $\mathrm{O} 1$ & 123.70 & 122.07 \\
O2 & $\mathrm{N}$ & $\mathrm{C} 1$ & 119.09 & 117.04 \\
O1 & $\mathrm{N}$ & $\mathrm{C} 1$ & 117.20 & 117.03 \\
\hline
\end{tabular}


Table 4. Torsion angles $\left({ }^{\circ}\right)$ by X-ray and theoretical calculations (B3LYP/6-31G*).

\begin{tabular}{cccccc}
\hline \multirow{2}{*}{ Atom1 } & \multirow{2}{*}{ Atom 2 } & \multirow{2}{*}{ Atom 3} & \multirow{2}{*}{ Atom $\mathbf{4}$} & \multicolumn{2}{c}{ Angle $\left({ }^{\circ}\right)$} \\
\cline { 5 - 6 } & & & & X-ray & B3LYP/6-31G* \\
\hline C4 & $\mathrm{C} 3$ & $\mathrm{C} 2$ & $\mathrm{C} 1$ & -1.07 & 0.003 \\
$\mathrm{C} 5$ & $\mathrm{C} 6$ & $\mathrm{C} 1$ & $\mathrm{C} 2$ & 0.92 & -0.015 \\
$\mathrm{C} 4$ & $\mathrm{C} 3$ & $\mathrm{C} 2$ & $\mathrm{C} 1$ & 0.08 & 0.011 \\
$\mathrm{H} 2$ & $\mathrm{C} 2$ & $\mathrm{C} 3$ & $\mathrm{C} 4$ & 178.76 & 179.99 \\
$\mathrm{H} 6$ & $\mathrm{C} 6$ & $\mathrm{C} 1$ & $\mathrm{C} 2$ & -179.28 & -180.00 \\
$\mathrm{H} 5$ & $\mathrm{C} 5$ & $\mathrm{C} 6$ & $\mathrm{C} 1$ & 178.63 & 179.98 \\
$\mathrm{H} 4$ & $\mathrm{C} 4$ & $\mathrm{C} 3$ & $\mathrm{C} 2$ & -179.68 & -179.99 \\
$\mathrm{O}$ & $\mathrm{C} 3$ & $\mathrm{C} 4$ & $\mathrm{C} 5$ & -179.68 & -179.97 \\
$\mathrm{H}$ & $\mathrm{O}$ & $\mathrm{C} 3$ & $\mathrm{C} 4$ & -6.70 & -179.98 \\
$\mathrm{~N}$ & $\mathrm{C} 1$ & $\mathrm{C} 2$ & $\mathrm{C} 3$ & -179.84 & 0.011 \\
$\mathrm{O} 1$ & $\mathrm{~N}$ & $\mathrm{C} 1$ & $\mathrm{C} 2$ & 179.21 & 179.84 \\
$\mathrm{O} 2$ & $\mathrm{~N}$ & $\mathrm{C} 1$ & $\mathrm{C} 2$ & 0.39 & 0.020 \\
\hline
\end{tabular}

\subsection{Electron Density Maps}

Figure 3 provides a comparison of the experimental static charge density of the molecule, obtained by convolution of the thermal motion from the charge density on the different atoms in the mean molecular plane, with the theoretical charge density, determined from a wave function for a pseudo atoms from an $a b$ initio calculation performed with a Gaussian basis setusing the Density Functional Theory at B3LYP level of theory at 6-31G*. As it can be seen, the two maps show reasonable agreement. These maps confirm the high quality of the data sets and the efficiency of the formalism of data processing as proposed by Blessing [18].

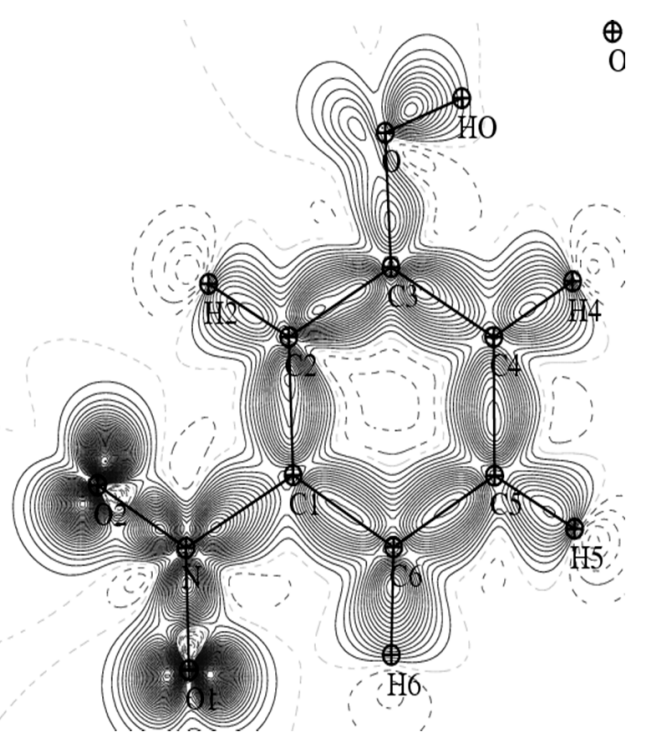

(a)

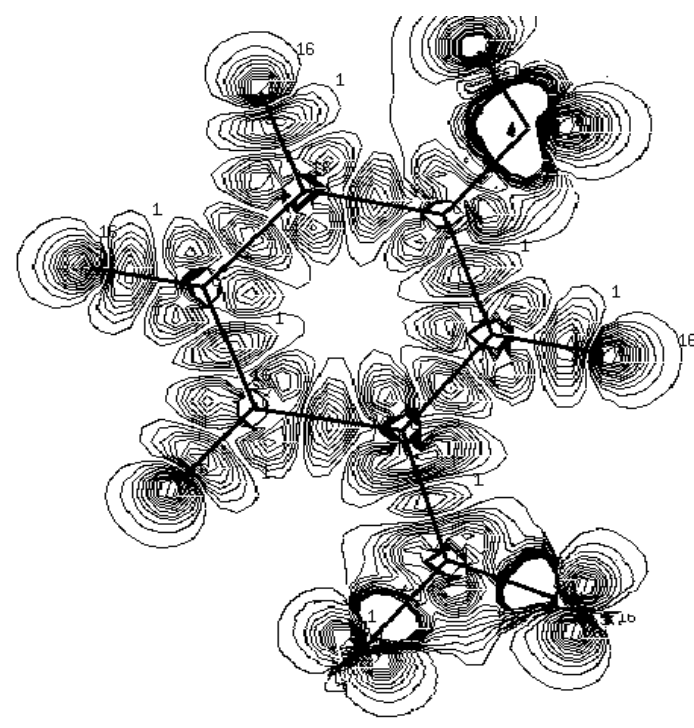

(b)

Figure 3. Comparison of the static and theoretical density maps of m-NPH. (a) Static density map. (b) Theoretical electron density map. 


\subsection{Net Atomic Charges}

Thevalence and mulitipolarpopulationceofficients were used to estimate the partial charges on the different atoms and the molecular dipole moment following the procedure described by Hansen and Coppens [8]. The experimantal net atomic charges have been previously published in an article about the high resolution X-ray diffraction and crystallographic study of m-NPH [5]. These values arecompared to the natural population analysis (NPA) charges derived from the ab initio calculations using B3LYP with the 6-31G* basis set (see Table 5, Figure 4). All the methods are in agreement for the evaluation of the positive sign of the net charges on the $\mathrm{H}$ and $\mathrm{N}$ atoms and the negative net charges on the $\mathrm{O}$ atoms.

Table 5. Atomiccharge of $m$-nitrophenol.

\begin{tabular}{ccc}
\hline Atom & Multipolar Refinement & B3LYP/6-31G* \\
\hline C1 & -0.1536 & 0.07099 \\
C2 & -0.2703 & -0.26992 \\
C3 & 0.0288 & 0.33201 \\
C4 & -0.3958 & -0.29354 \\
C5 & -0.4621 & -0.21591 \\
C6 & -0.2689 & -0.24740 \\
N & 0.6466 & 0.51462 \\
O1 & -0.2337 & -0.38131 \\
O2 & -0.2189 & -0.38013 \\
O & -0.2901 & -0.68228 \\
H2 & 0.2187 & 0.28515 \\
H4 & 0.2165 & 0.24054 \\
H5 & 0.2508 & 0.25207 \\
H6 & 0.2885 & 0.27564 \\
H & 0.3289 & 0.49644 \\
\hline
\end{tabular}

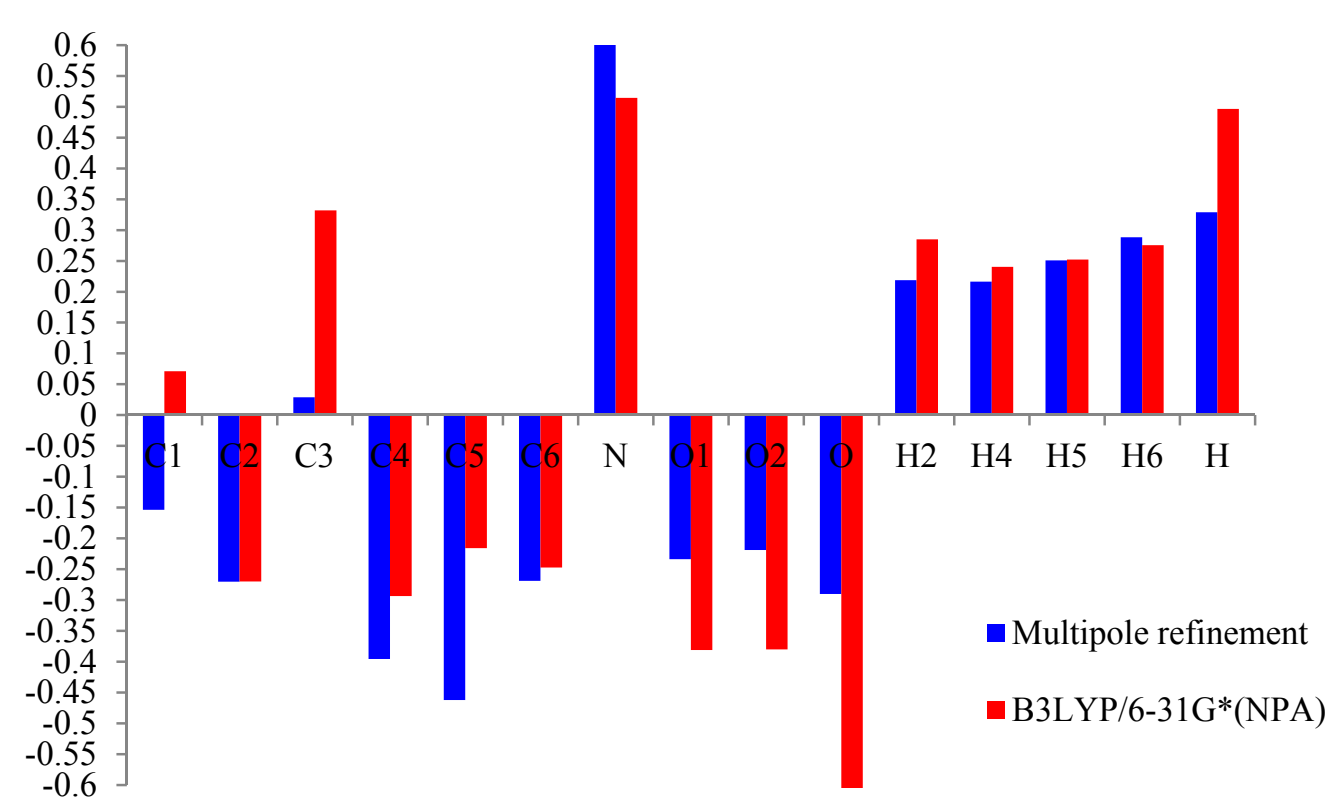

Figure 4. Histogram of the value of the net atomic charge in both methods multipolar refinement and B3LYP of $m$-nitrophenol. 


\subsection{Molecular Moments}

From the knowledge of the density function one can derive some important physical properties of the molecules such as the surrounding electrostatic field gradient, and the different electrostatic moments of the charge distribution [14]. A property associated to the average value of a quantum observable $\langle O\rangle$ is linked to the density function as given by the general equation (3), $V$ is the molecular volume:

$$
\langle O\rangle=\int_{V} \hat{O}(\vec{r}) \rho(\vec{r}) d \vec{r}
$$

If $\Delta \rho(\vec{r})$ rather than $\rho(\vec{r})$ is being considered the electrostatic moment due to the deformation density in the molecule and can be estimated. The experimental molecular dipole moment of m-NPH has been determined in the previous paper cited above using the multipolar model [5]. Such studies have clearly evidenced the electron donor character of the $\mathrm{C}-\mathrm{H}$ groups in conjunction with the electron acceptor character of the nitro and hydroxyl groups. In general, the experimental method provides a magnitude of about 5.80 Debye for the dipole moment. A theoretical calculation has been performed usingB3LYP at 6-31G* basis set in order to carried out the components of the molecular dipole moment. The obtained results are summarized in Table 6 in which the experimental values are given for comparison. The orientation of the different vectors of dipole moment in the molecular axial system is shown in Figure 5.

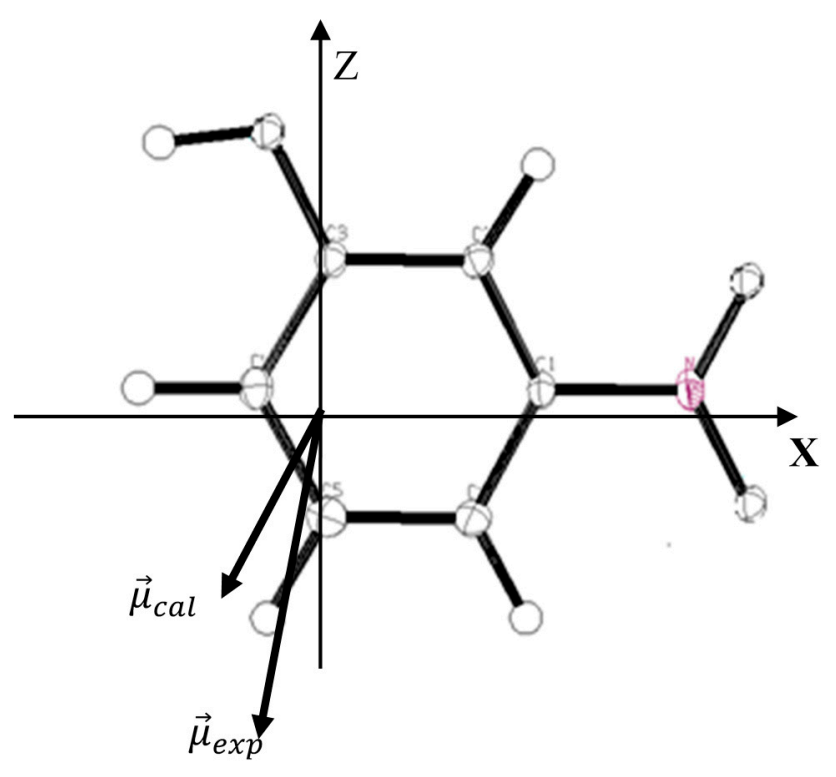

Figure 5. Orientation of the molecular dipole moment of m-NPH: $\vec{\mu}_{\text {exp }}$ : molecular dipole moment from the experimental study; $\vec{\mu}_{c a l}$ : molecular dipole moment from the theoretical DFT calculations. 
Table 6. Components of the molecular dipolar moment from DFT calculations (B3LYP at 6-31G* basis set) and X-ray experiment. The origin coincides with the center of mass of the molecule, and the Cartesian system referred to the inertial axis of the molecule.

\begin{tabular}{cccccc}
\hline Methods & Models & $\mu_{X}$ & $\mu_{Y}$ & $\mu_{Z}$ & $\mu$ Debye \\
\hline X-ray Experiment & Multipolar refinement & -0.3209 & -0.3200 & -6.3358 & 5.8000 \\
Ab initio & DFT(B3LYP/6-31G*) & -2.1194 & -0.0010 & -5.4234 & 5.8228 \\
\hline
\end{tabular}

The components of the electrostatic quadrupole moment are obtained by substituting in Equation (3) the operator $\hat{O}(r)$ by $\vec{r}_{\alpha} \Lambda \vec{r}_{\beta}$. If in that equation the density function $\rho(\vec{r})$ is replaced by the multipolar expansion up to order $l=1$, then the components of the quadrupole moment are given by:

$$
Q_{\alpha \beta}=\sum\left[Q_{\alpha \beta}^{i}+r_{i \beta} d_{i \alpha}+r_{i \alpha} d_{i \beta}+r_{i \alpha} r_{i \beta} q_{i}\right]
$$

where $d_{i \alpha}$ and $q_{i}$ represent respectively the component of the dipole moment and the net charge of atom i at $r_{i} \cdot Q_{\alpha \beta}^{i}$ are the atomic quadrupoles neglected here.

In the case of the direct integration method the development of Equation (3) leads to:

$$
Q_{\alpha \beta}=\frac{1}{V} \sum_{\vec{H}} \Delta F(\vec{H})\left[\sum_{i}\left(Q_{\alpha \beta}^{i}+r_{i \beta} d_{i \alpha}+r_{i \alpha} d_{i \beta}+r_{i \alpha} r_{i \beta} q_{i}\right)\right]
$$

with:

$$
Q_{\alpha \beta}^{i}=\int_{t_{i}}\left(r_{\alpha}-r_{i}\right)\left(r_{\beta}-r_{i}\right) e^{i 2 \pi \vec{H}\left(\vec{r}-\vec{r}_{i}\right)} d^{3} r
$$

The summation over $\vec{H}$ is performed over all structure factors and the indice $t_{i}$ designates the integrable subunits. Evaluation of all molecular moments requires summations of the density and moments of each subunit which are being performed according to a space partitioning scheme. The quadrupolar moment values are reported in the Table 7 with the analogous components obtained from the point charge model using the net atomic charges derived by NPA method calculations. The most remarkable features when comparing experimental values with those derived from the free molecule stand-out in the $Q_{X X}, Q_{Z Z}$ and $Q_{X X}$ components. The experimental second moment component relative to a chosen molecular origin, $\left(Q_{X X}=-55.53, Q_{z Z}=-63.88\right)$ shows a weaker charge expansion than in the free molecule $\left(Q_{X X}=-53.63, Q_{Z Z}=-51.53\right)$ while the positive $Q_{X Z}$ 's indicate a similar contraction in the $(\vec{X}+\vec{Z})$ direction (orientations in the molecular frame given in Figure 5) for both the free molecule and the molecule in the crystal state. On the other hand the same electronic delocalization in the $(\vec{X}+\vec{Z})$ direction is being observed in the molecular plane for molecules in both states.

Table 7. Components of the molecular quadrupole moment of the charge distribution (e. $\left.\AA^{2}\right)$ from theoretical calculations and experimental electron density study.

\begin{tabular}{ccc}
\hline Quadrupole Moments & X-ray Experiment & Ab Initio DFT(6-31G) \\
\hline$Q_{X X}$ & -55.532 & -53.632 \\
$Q_{Y Y}$ & -53.129 & -53.777 \\
$Q_{Z Z}$ & -63.886 & -51.536 \\
\hline
\end{tabular}


Table 7. Cont.

\begin{tabular}{ccc}
\hline Quadrupole Moments & X-ray Experiment & Ab Initio DFT(6-31G) \\
\hline$Q_{X Y}$ & -1.825 & 0.964 \\
$Q_{X Z}$ & 3.878 & 0.002 \\
$Q_{Y Z}$ & -1.755 & -0.001 \\
\hline
\end{tabular}

\subsection{Frontier Molecular Orbital Analysis}

Molecular orbitals (HOMO-LUMO) and their properties such as energy are very useful for physicist and chemists and are very important parameters for quantum chemistry. This is also used by the frontier electron density for predicting the most reactive position in $\pi$-electron systems and also explains several types of reaction in conjugated system [19]. The conjugated molecules are characterized by a small highest occupied molecular orbital- lowest unoccupied molecular orbital (HOMO-LUMO) separation. Both the highest occupied molecular orbital and lowest unoccupied molecular orbital are the main orbitals which take part in chemical stability. The HOMO represents the ability to donate an electron, LUMO as an electron acceptor, represents the ability to obtain an electron. The HOMO and LUMO energy calculated by B3LYP/6-311++G(d,p) method is shown below. This electronic absorption corresponds to the transition from the ground to the first excited state and is mainly described by one electron excitation from the highest occupied molecular orbital to the lowest unoccupied molecular orbital. While the energy of the HOMO describe the ionization potential, LUMO energy is concerned by the electron affinity Energy difference between HOMO and LUMO orbital is called as energy gap which is an important stability for structures and is calculated as:

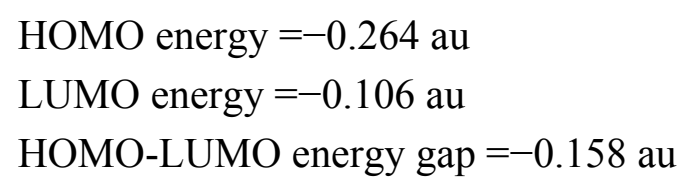

It has been shown that calculated energy gap between HOMO and LUMO can be very useful to prove the activity from intramolecular charge transfer [20].

\subsection{Electrostatic Potential}

In order to grasp the molecular interactions, the molecular electrostatic potential (MEP) is used. The molecular electrostatic potential is the potential that a unit positive charge would experience at any point surrounding the molecule due to the electron density distribution in the molecule. The electrostatic potential is considered predictive of chemical reactivity because regions of negative potential are expected to be sites of protonation and nucleophilic attack, while regions of positive potential may indicate electrophilic sites. The distribution of the electrostatic potential for the molecule in the crystal was calculated from Equation (7):

$$
\Phi(r)=\int \frac{\rho_{\text {total }}(r)}{\left|r-r^{\prime}\right|} d r
$$

where $\rho_{\text {total }}$ represents both the nuclear and the electronic charge. The integration is over the molecular volume, and $r^{\prime}$ represents the atomic position relative to same origin. The integration includes the atoms 
of only one molecule and therefore does not include directly the effects of charge distribution of the molecules.

Figure 6 shows the experiment and theoretical maps of the electrostatic potential distribution in the plane of the base ring. We are used the Density Functional Theory at B3LYP level of theory at 6-31G* to describe the theoretical electrostatic potential map. Figure 7 is the same representation in $3 \mathrm{D}$ dimensions of the theoretical electrostatic potential map. The extension of the positive electrostatic potential around the $\mathrm{C}-\mathrm{H}$ group and the regions of negative electrostatic potential around the nitro and hydroxyl group gives same conclusion about the nature of the intramolecular charge transfer as found by the orientation of the molecular dipole moment.

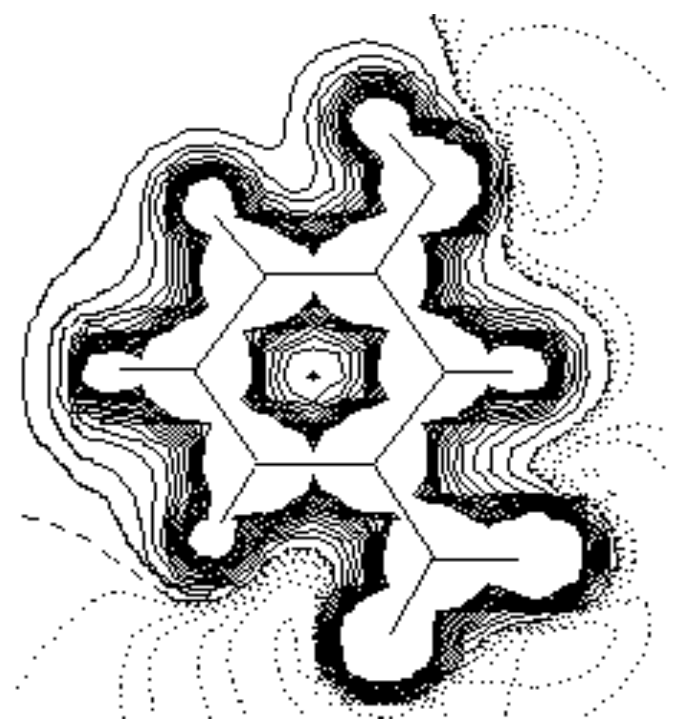

(a)

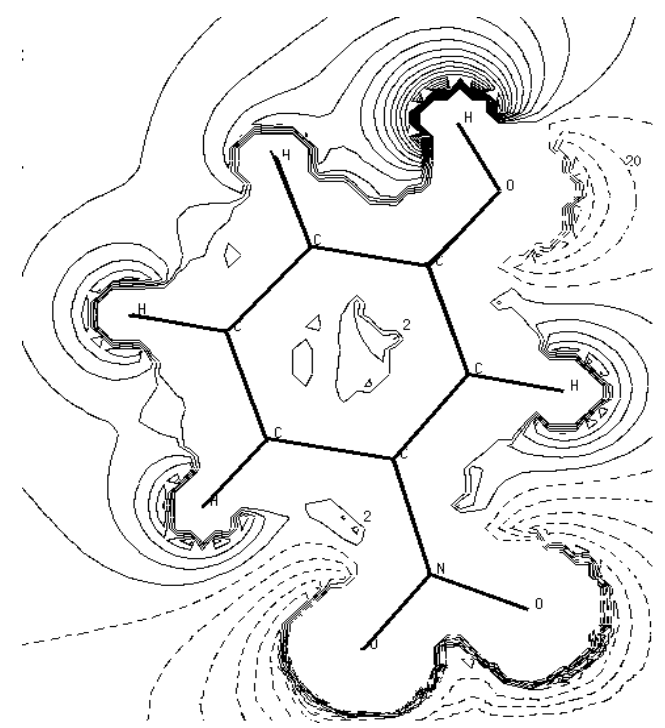

(b)

Figure 6. The electrostatic potential maps around the molecule. The section is in the plane of the ring atoms. (a) Experimental (contours are at $0.05 \mathrm{e}^{-1}$ ). (b) Theoreticalusing the Density Functional Theory at B3LYP level of theory at 6-31G* (contours are at 0.025 e $\AA^{-1}$ ). Zero and negative contours are dashed lines $\left(1 \mathrm{e} \AA^{-1}=332.1 \mathrm{kcal} \cdot \mathrm{mol}^{-1}\right)$.

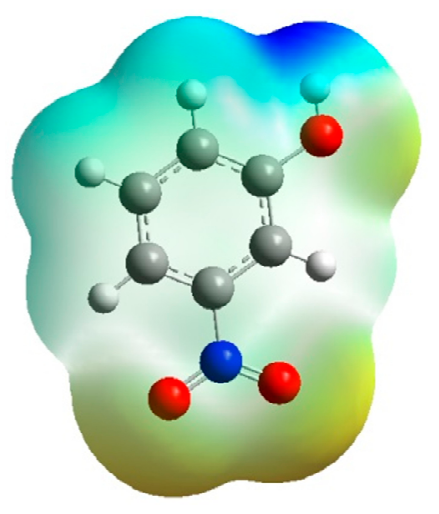

Figure 7. 3D-representation of the electrostatic potential around the molecule using the Density Functional Theory at B3LYP level of theory at 6-31G*

The potential of the $m$-nitrophenol molecule has been calculated from the experimental electron density distribution by the multipolar method using the X-ray diffraction data. The comparison of the experimental 
potential in a crystal and the theoretical potential for an isolated molecule is an excellent test for high quality descriptive model for the electron charge density distribution from X-ray diffraction experiment.

\section{Conclusions}

In this article, we have dealt with the salient features of the electronic charge density distribution in molecular solids obtained by both theory and experiment. This study has obtained good accurate results on the structure and electron charge density which back the experimental results for the electron charge density distribution.

The general conclusion from the estimation of the dipolar moments and the electrostatic potential of the $m$-nitrophenol molecule in the both experimental and theoretical study is that the region of the nitro and hydroxyl groups is electronegative and the $\mathrm{C}-\mathrm{H}$ group region is electropositive. These results could be used to explain the existence of the polymorphism in $m$-nitrophenol compounds, if they were completed by the study of the nature and the energy of the molecular interaction by the X-ray diffraction of the both polymorphic of m-NPH.

\section{Acknowledgments}

Thanks are due to MESRS (Ministère de l'Enseignement Supérieur et de la Recherche Scientifique Algérie) for financial support via the CNEPRU program.

\section{Author Contributions}

Mokhtaria Drissi designed research, performed the crystallographic and the theoretical studies, discussed the results and wrote the manuscript. Nadia Benhalima, Youcef Megrouss, Rahmani Rachida contributed to molecular modeling studies and discussed results; Abdelkader Chouaih and Fodil Hamzaoui contributed with literature research performed the experiments, analyzed the data, discussed results and wrote the paper.

\section{Conflicts of Interest}

The authors declare no conflict of interest.

\section{References}

1. Panadares, F.; Ungaretti, L.; Coda, A. The crystal structure of a monoclinic phase of $m$-nitrophenol. Acta Cryst. B 1975, 31, 2671-2675.

2. Wojcik, G.; Toupet, L. The inter-and intramolecular charge transfer along the polymeric chain of hydrogen-bonded molecules in two crystal forms of $m$-nitrophenol. Mol. Cryst. Liquid Cryst. 1993, 229, 153-159.

3. Hamzaoui, F.; Baert, F.; Wojcik, G. Electron-density study of $m$-nitrophenol in theorthorhombic structure. Acta Cryst. B 1996, 52, 159-164.

4. Wojcik, G.; Marqueton, Y. The phase transition of m-nitrophenol. Mol. Cryst. Liquid Cryst. 1989, $168,247-254$. 
5. Hamzaoui, F.; Drissi, M.; Chouaih, A.; Lagant, P.; Vergoten, G. Electron Charge Density Distribution from X-ray diffraction Study of the M-Nitrophenol compound in the monoclinic form. Int. J. Mol. Sci. 2007, 8, 103-115.

6. Wojcik, G.; Mossakowska, G. Polymorphs of $p$-nitrophenol as studied by variable temperature X-ray diffraction and calorimetry: Comparison with $m$-nitrophenol. Acta Crystallogr. 2006, B62, 143-152.

7. Wojcik, G. Intermolecular interactions leading to crystal polymorphism of organic compounds. X-ray diffraction and quantum chemical studies of para- and meta-Nitrophenol. Ser. Khim. 2007, 48, 123-127.

8. Jeffrey, G.A.; Cruickshank, D.W.J. Molecular structure determination by X-ray crystal analysis: Modern methods and their accuracy. Q. Rev. Chem. Soc. 1953, 7, 335-376.

9. Coppens, P.; Volkov, A. The interplay between experiment and theory in charge-density analysis. Acta Crystallogr. 2004, A60, 357-364.

10. Hansen, N.K.; Coppens, P. Testing aspherical atom refinements on small-molecule datasets. Acta Crystallogr. 1978, A34, 909-921.

11. Jelsch, C.; Guillot, B.; Lagoutte, A.; Lecomte, C. Advances in protein and small-molecules charge-density refinement methods using MoPro. J. Appl. Crystallogr. 2005, 38, 38-54.

12. Volkov, A.; Abramov, Y.; Coppens, P.; Gatti, C. On the origin of topological differences between experimental and theoretical crystal charge densities. Acta Crystallogr. 2000, A56, 332-339.

13. Volkov, A.; Gatti, C.; Abramov, Y.; Coppens, P. Evaluation of net atomic charges and atomic and molecular electrostatic moments through topological analysis of the experimental charge density. Acta Crystallogr. 2000, A56, 252-258.

14. Coppens, P. X-ray Charge Densities and Chemical Bonding; Oxford University Press: New York, NY, USA, 1997.

15. Hehre, W.J.; Schleyer, R.R.; Pople, J.A. Ab Initio Molecular Orbital Theory; John Wiley \& Sons: Hoboken, NJ, USA, 1986.

16. Frisch, J.; Trucks, G.W.; Schlegel, H.B.; Scuseria, G.E.; Robb, M.A.; Cheeseman, J.R.; Montgomery, J.A., Jr.; Vreven, T.; Kudin, K.N.; Burant, J.C.; et al. Gaussian 03, Revision A.1; Gaussian, Inc.: Pittsburgh, PA, USA, 2003.

17. Schaftenaar, G.; Noordik, J.H. Molden: A pre- and post- processing program for molecular and electronic structures. J. Comput.-Aided Mol. Des. 2000, 14, 123-134.

18. Blessing, R.H. DREAD - data reduction and error analysis for single-crystal diffractometer data. J. Appl. Crystallogr. 1989, 22, 396-397.

19. Choi, C.; Kertez, M. Conformational information from vibrational spectra of styrene, trans-stilbene, and cis-stilbene. J. Phys. Chem. 1997, 101A, 3823-3831.

20. Padmaja, L.; Ravi Kumara, C.; Sajan, D.; Joe, I.H.; Jayakumar, V.S.; Pettit, G.R.; Nielsen, O.F. Density functional study on the structural conformations and intramolecular charge transfer from the vibrational spectra of the anticancer drug combretastatin-A2. J. Raman Spectrosc. 2009, 40, 419-428.

Sample Availability: Samples of the compounds are available from the authors.

(C) 2015 by the authors; licensee MDPI, Basel, Switzerland. This article is an open access article distributed under the terms and conditions of the Creative Commons Attribution license (http://creativecommons.org/licenses/by/4.0/). 